\title{
PERCEPCIONES QUE TIENEN TRABAJADORES CHILENOS SOBRE EL IMPACTO DEL TELETRABAJO EN EL ENTORNO DE COVID-19
}

\author{
PERCEPTIONS OF CHILEAN WORKERS ON THE IMPACT OF \\ TELEWORKING IN THE CONTEXT OF COVID-19
}

\section{PERCEPÇÓES QUE OS TRABALHADORES CHILENOS TÊM SOBRE O IMPACTO DO TELETRABALHO NO AMBIENTE COVID-19}

\author{
Francisca Céspedes Hernández* \\ Claudia Fuentes Marchant** \\ Valeria Molina Pradenas ${ }^{* * *}$ \\ Catalina Rebolledo Hernández**** \\ Carolina Luengo Martínez***** \\ Sergio Madero Gómez ${ }^{* * *}$
}

\begin{abstract}
RESUMEN
La pandemia ha generado diversos cambios que han llevado a las empresas a buscar estrategias para continuar sus procesos productivos y proteger la salud de los trabajadores; entre estas, la más destacada es el Teletrabajo. Objetivo: Analizar las percepciones que tienen los trabajadores chilenos sobre el impacto del teletrabajo en términos de Estrés laboral, Balance Vida-Trabajo y Satisfacción, en ambiente de contingencia producto del COVID-19. Material y Método: Investigación de tipo cuantitativa, tipo relacional y de corte transversal que utilizó un muestreo por conveniencia y se obtuvieron datos de 225 trabajadores chilenos que estaban realizando teletrabajo producto del COVID-19 y respondieron la encuesta on-line. Se utilizó un instrumento con 26 reactivos, que se aplicó a trabajadores mayores de edad que estuvieran realizando actividades desde casa, entre los meses de septiembre a octubre de 2020, logrando obtener 181 respuestas útiles. Resultados: Los trabajadores
\end{abstract}

*Licenciada en Enfermería, Departamento Enfermería, Facultad de Ciencias de la Salud y de Los Alimentos, Universidad del BíoBío, Chillán, Chile. ORCID: https://orcid.org/0000-0002-6992-0038 Email: fran.cespedess@gmail.com

**Licenciada en Enfermería, Departamento Enfermería, Facultad de Ciencias de la Salud y de Los Alimentos, Universidad del Bío-Bío, Chillán, Chile. ORCID: https://orcid.org/0000-0003-1394-4126 Email: clauu.fer@hotmail.com

*** Licenciada en Enfermería, Departamento Enfermería, Facultad de Ciencias de la Salud y de Los Alimentos, Universidad del Bío-Bío, Chillán, Chile. ORCID: https://orcid.org/0000-0001-9300-0074 Email: valeria.molina1701@alumnos.ubiobio.cl

****Licenciada en Enfermería, Departamento Enfermería, Facultad de Ciencias de la Salud y de Los Alimentos, Universidad del Bío-Bío, Chillán, Chile. ORCID: https://orcid.org/0000-0001-9210-4584 Email: catalinarebolledoh@gmail.com

*****Enfermera, Doctora en Enfermería, Académica Departamento Enfermería, Facultad de Ciencias de la Salud y de Los Alimentos, Universidad del Bío-Bío, Chillán, Chile. ORCID: https://orcid.org/0000-0002-6541-3645 Email: cluengo@ubiobio. cl Autora de correspondencia

******Licenciado en Sistemas Computacionales, Doctor en Economía y Dirección de Empresas, Académico Departamento de Gestión y Liderazgo, Escuela de Negocios, Tecnológico de Monterrey, Monterrey, NL. México. ORCID: https://orcid.org/00000003-3996-7609 Email: smadero@tec.mx Autor de correspondencia 
que se encuentran en modalidad telemática corresponden mayormente a mujeres, solteras, entre 24 a 40 años, del ámbito educativo, del sector público y tienen experiencia laboral mayor a diez años; existe una relación positiva entre la satisfacción laboral y el teletrabajo $(r=0,381)$, mientras que para las variables Estrés laboral y Balance vida-trabajo sucede lo contrario, es decir, una relación negativa $(r=-0,408)$. Conclusión: Las actividades telemáticas producen a los trabajadores sintomatología de estrés, como tensión y/o ansiedad, pero a pesar de esto, tienen una actitud positiva ante este cambio en la modalidad laboral, tratando de vivir el día a día y buscando cómo apoyar a otros.

Palabras clave: Teletrabajo; Estrés laboral; Equilibrio entre Vida Personal y Laboral; COVID-19.

\begin{abstract}
The pandemic has caused several changes that have led companies to look for strategies to continue their production processes and protect the health of workers. Among these strategies, the most important is Teleworking. Objective: To analyze the perceptions that Chilean workers have on the impact of teleworking in terms of work stress, work-life balance and satisfaction, in a contingency environment as a result of COVID-19. Material and Method: Quantitative, relational and cross-sectional research using convenience sampling and data from 225 Chilean workers who were teleworking as a result of COVID-19 and responded to the on-line survey. An instrument with 26 items was applied to workers of legal age who were carrying out activities from home, between the months of September to October 2020, collecting 181 useful responses. Results: People working from home correspond mostly to single women, between 24 to 40 years old, who work in the field of education or the public sector and have work experience of more than ten years; there is a positive relationship between job satisfaction and teleworking $(\mathrm{r}=0.381)$, while for the variables work stress and work-life balance a negative relationship was observed $(\mathrm{r}=-0.408)$. Conclusion: Teleworking produces stress symptoms among workers, such as tension and/or anxiety. Nevertheless, workers have a positive attitude towards this modality, trying to adapt and seeking support from other people.
\end{abstract}

Key words: Teleworking; Occupational Stress; Work-Life Balance; COVID-19.

\title{
RESUMO
}

A pandemia gerou várias mudanças que levaram as empresas a procurar estratégias para continuar os seus processos de produção e proteger a saúde dos trabalhadores; entre estas, a mais relevante é o Teletrabalho. Objetivo: Analisar as percepçóes dos trabalhadores chilenos sobre o impacto do teletrabalho em termos de Estresse no trabalho, Equilíbrio Vida-Trabalho e Satisfação, em um ambiente de contingência resultante da COVID-19. Material e Método: Pesquisa quantitativa, do tipo relacional e corte transversal utilizando amostragem de conveniência e obtendo dados de 225 trabalhadores chilenos que estavam realizando teletrabalho como resultado da COVID-19 e responderam ao questionário online. Foi utilizado um instrumento com 26 reativos, que foi aplicado a trabalhadores adultos que realizavam atividades desde casa, entre setembro e outubro de 2020, obtendo 181 respostas úteis. Resultados: Os trabalhadores que estão no modo telemático, correspondem maioritariamente a mulheres, solteiras, entre 24 e 40 anos, do campo educativo, do sector público e têm mais de dez anos de experiência de trabalho; existe uma relação positiva entre a satisfação profissional e o teletrabalho $(\mathrm{r}=0,381)$, enquanto para as variáveis Estresse no trabalho e Equilíbrio Vida-Trabalho acontece o contrário, ou seja, uma relaçáo negativa $(r=-0,408)$. Conclusão: As atividades telemáticas produzem sintomas de estresse nos trabalhadores, tais como tensão e/ou ansiedade, mas apesar disso, têm uma atitude positiva em relaçáo a esta mudança na modalidade de trabalho, tentando viver dia após dia e procurando formas de apoiar os outros.

Palavras-chave: Teletrabalho; Estresse Ocupacional COVID-19; Equilíbrio Trabalho-Vida.

Fecha de recepción: 12/03/2021

Fecha de aceptación: 06/05/2021 


\section{INTRODUCCIÓN}

La pandemia COVID-19 es uno de los desafíos más serios que ha enfrentado la humanidad en tiempos recientes ${ }^{(1)}$. Este crecimiento acelerado de los casos confirmados y de los fallecimientos también se extendió por todo el mundo ${ }^{(2)}$. Esta pandemia dio lugar a muchos cambios en el convivir diario y uno de los principales tiene que ver con la cuarentena o aislamiento, en donde las personas y sus familias permanecen en sus casas para no estar expuestos al contagio del virus ${ }^{(3)}$.

Estas medidas de confinamiento de la población ha llevado a las empresas a buscar estrategias para continuar sus procesos productivos y proteger la salud de los trabajadores, entre estas, la más destacada es el Teletrabajo (TET). Datos nacionales reportan que entre un $15 \%$ y un $20 \%$ de los trabajadores en la Región Metropolitana están operando bajo algún tipo de modalidad de trabajo virtual $^{(4)}$, mientras que en regiones esta modalidad, aun cuando está operando de la misma forma, es en porcentajes menores.

El trabajo desde el hogar (working from home) o teletrabajo, ha facilitado la continuidad de muchas actividades productivas, con el consiguiente efecto positivo sobre el mantenimiento del empleo ${ }^{(4)}$. El término Teletrabajo se acuñó por primera vez en la década de 1970 y, actualmente, se define como la actividad laboral que se desarrolla fuera de las instalaciones de la empresa, con el uso de Tecnologías de la Información y de la Comunicación (TIC), en el que el teletrabajador es la persona que utiliza la telemática para la realización de su profesión ${ }^{(5)}$.

Ahora, dado el rotundo cambio de modalidad presencial a teletrabajo por la pandemia, la Ley Número 21.220 modifica el Código del Trabajo chileno en materia de trabajo a distancia, definiendo como trabajo a distancia, aquel en el que el trabajador presta sus servicios, total o parcialmente, desde su domicilio u otro lugar o lugares distintos de los establecimientos, instalaciones o faenas de la empresa. Se denominará teletrabajo si los servicios son prestados mediante la utilización de medios tecnológicos, informáticos o de telecomunicaciones ${ }^{(6)}$.

De acuerdo a Lampert y Poblete ${ }^{(7)}$, esta modalidad de trabajo puede tener tanto beneficios como perjuicios para el trabajador, dependiendo de factores, tales como: personales, relación con las jefaturas, grado de autonomía, flexibilidad horaria, características del lugar donde se desarrolla, calidad y grado de manejo de la TIC que se tengan a disposición, conectividad y tipo de contrato. Al respecto, Tavares ${ }^{(8)}$ asegura que esta modalidad impacta de manera positiva en el aspecto individual, ya que la flexibilidad permite que los teletrabajadores puedan resolver, paralelamente, problemas dentro de su hogar y, a la vez, realizar actividades recreativas. Relevándose un impacto positivo en la conciliación entre vida laboral, familiar y personal, la flexibilidad y autonomía del trabajo telemático otorga la libertad para planificar, organizar tareas, administrar los tiempos para el trabajo autónomo y ahorrar el tiempo de traslado $^{(3,5)}$.

Aun así, hay autores que señalan que no todos los trabajadores son capaces de armonizar trabajo y familia, ya que el no separar ambos mundos puede llevar a la excesiva injerencia de las responsabilidades familiares en el trabajo de la persona, por asumir más tareas domésticas de las que puede realizar para el correcto desempeño de su trabajo ${ }^{(9)}$.

En este contexto, el Balance Vida-Trabajo (BVT) es considerado como la relación dinámica entre el tiempo y espacio que dedica un individuo a su trabajo y a su vida no laboral ${ }^{(10)}$ y ha tomado mayor importancia en los últimos años ${ }^{(11)}$, dado que puede llevar a un conflicto entre la vida laboral y familiar (CTF), definido por Greenhaus y Beutell ${ }^{(12)}$ como una "forma de conflicto de rol, en el que las presiones que resultan del trabajo y las presiones familiares son mutuamente incompatibles en algún aspecto", "la presión de los roles que se asumen en el trabajo y la familia son, de alguna forma mutuamente incompatibles[sic]", afectando el bienestar, el funcionamiento de las organizaciones, relaciones sociales, familiares y laborales. El CTF surge principalmente por la excesiva demanda de tiempo en el trabajo, incompatibilidad en los horarios y la tensión causada por los intentos de la persona para cumplir con los deberes que se desprenden del trabajo y de la familia ${ }^{(13)}$.

En efecto, la modalidad de teletrabajo presenta algunas desventajas a nivel ergonómico, psicosocial, aislamiento y la difícil separación de la vida familiar y laboral ${ }^{(5)}$. Por ello, Bae y Yang $^{(14)}$ mencionan que el teletrabajo no aumenta la satisfacción laboral debido a la reducción de interacción social. Sumado a esto, se ha identificado aumento de estrés 
en los trabajadores, el cual se ha convertido en un problema prevalente en el ámbito laboral ${ }^{(15)}$ y ha sido definido como las "reacciones nocivas físicas y emocionales que ocurren cuando las exigencias del trabajo no igualan las capacidades, los recursos o las necesidades del trabajador" ${ }^{(15)}$, ocasionando alteraciones a nivel psicosomático, conductual y emocional ${ }^{(16)}$.

Análogamente, Soto et al. ${ }^{(17)}$ señalan que el teletrabajo ha afectado negativamente algunas dimensiones relevantes de la calidad de vida en el trabajo, como un aumento de carga laboral que podría explicarse con los procesos de adaptación de los teletrabajadores. Por lo demás, este cambio tan repentino en la modalidad laboral no ha permitido a los trabajadores, en su mayoría, a planificar las condiciones, tanto físicas, tecnológicas como organizacionales. Todas estas exigencias combinadas pueden hacer que la persona que realice teletrabajo manifieste problemas físicos (dolores, lesiones musculoesqueléticas, problemas visuales, cefalea, etc.) y mentales (estrés, ansiedad, irritabilidad, insatisfacción), entre otros ${ }^{(18)}$.

De esta manera, el presente trabajo tiene como objetivo analizar las percepciones que tienen los trabajadores chilenos sobre el impacto del teletrabajo en términos de Estrés laboral, Balance Vida-Trabajo y Satisfacción, en un ambiente de contingencia producto del COVID-19, así como medir la validez y confiabilidad del instrumento de medida.

\section{MATERIAL Y MÉTODO}

Para el desarrollo de esta investigación de tipo cuantitativo, analítico y de corte transversal, se utilizó un muestreo por conveniencia y se obtuvieron los datos de 225 trabajadores chilenos, que estaban realizando teletrabajo, producto del COVID-19 y respondieron la encuesta on-line. No obstante, se utilizaron los datos sólo de 181 trabajadores $(80,4 \%)$ que cumplieron el requisito de realizar exclusivamente teletrabajo en este período además de ser mayores de edad.

El período comprendido para la aplicación del cuestionario fue entre el 28 de septiembre al 29 de octubre de 2020, fechas en las que en el país estaba la indicación de la suspensión de actividades laborales de manera presencial y se tenían que realizar en la modalidad a distancia por causa del COVID-19. Aprovechando los recursos tecnológicos y debido al riesgo de contagio por el COVID-19, principalmente en las fechas en que se realizó el trabajo de campo, la convocatoria y acercamiento a los participantes fue a través de redes sociales de grupos profesionales asociados a diferentes áreas laborales (educación, servicios, salud, comercial, industrial), sumado a diversas personas de las redes de contacto de los autores.

Para el levantamiento de los datos se diseñó un cuestionario en línea, en el formato Google Forms, con 26 ítems, de los cuales 5 eran de antecedentes sociodemográficos, 3 de actividades laborales, y los 18 restantes fueron divididos en 4 constructos: 1) Estrés por el trabajo a distancia (EST), compuesto por 4 ítems tomados de Madero et al. ${ }^{(19)}$, Madero y Flores ${ }^{(20,21)}$ y adaptados por los autores, pues originalmente estaban en una escala diseñada para medir el nivel de estrés generado por la epidemia de la influenza N1H1 ocurrida en México durante el año 2009. En un estudio más reciente, realizado por Madero et al. ${ }^{(19)}$, el estrés percibido en el contexto del COVID-19 alcanzó un índice de fiabilidad de $0,85.2)$ Balance vida-trabajo (BVT), con 5 ítems, también adaptados por los autores para el contexto del COVID-19. 3) Teletrabajo (TET), con 5 ítems, también tomados de Madero et al. ${ }^{(19)} \mathrm{y}$ adaptados por los autores a partir de 6 ítems del documento original con un índice de fiabilidad de $0,84.4)$ Satisfacción en el trabajo, con 4 ítems, que también fueron revisados para que los diversos ítems estuvieran en el contexto del COVID-19. El formato de las escalas fue de tipo Likert, siendo 1 "totalmente en desacuerdo" y 5 "totalmente de acuerdo".

Para cumplir con el proceso de la validez del instrumento, se consultó a dos expertos en el tema de gestión de recursos humanos en las organizaciones y se aplicó una prueba piloto a un grupo de treinta personas, no incluidos en la muestra de estudio. Dicha prueba se realizó con la finalidad de conocer el tiempo utilizado en el llenado del instrumento, identificar posibles errores en la redacción de los diversos ítems y poder llegar a la versión final del instrumento de investigación. Después de las correcciones finales, nuevamente se consultó a los expertos, destacando que el instrumento diseñado cumplía con la validez y se podía iniciar la recolección de datos. 
Para realizar la recolección de datos se solicitó la autorización correspondiente del Comité de Ética de la Universidad del Bío-Bío, según Ley 20.120, artículo $10^{(22)}$. Una vez que se autorizó el trabajo de campo, la participación en este estudio fue de carácter voluntario. El cuestionario que fue enviado a los trabajadores incluía el consentimiento informado, el cual debía ser leído, comprendido y aprobado por el participante. Se mantuvo la confidencialidad en el manejo de la información entregada por los trabajadores en concordancia con la Ley $19.628^{(23)}$ y el Código Internacional de Nuremberg ${ }^{(24)}$. Durante toda la realización de este estudio se consideraron los principios éticos de Emanuel ${ }^{(25)}$.

En el control de calidad de los datos, se obtuvieron valores de alfa de Cronbach que indicaron una buena consistencia interna de los instrumentos $\left.^{(26)}: 1\right)$ Estrés por el trabajo a distancia (4 ítems $)=0,829 ; 2)$ Balance vida-trabajo (5 ítems $)=0,908$; 3) Teletrabajo (5 ítems) $=0,709 \mathrm{y}$ 4) Satisfacción en el trabajo ( 4 ítems) $=0,777$. Para el procesamiento de los datos se utilizó estadística descriptiva e inferencial, a través del software IBM SPSS, versión 24.

\section{RESULTADOS}

Características sociodemográficas y laborales de trabajadores en teletrabajo (Tabla 1): De los 181 trabajadores, el $76,2 \%$ era del género femenino, $55,8 \%$ soltera/o, 53\% nacidos entre 1981 al 1997 , el $55,2 \%$ con uno o más hijos y mayoritariamente $(60,2 \%)$ de la Región de Nuble. De acuerdo a las características laborales, se destaca que, mayoritariamente, los trabajadores se desempeñan en el ámbito educativo (37\%), pertenecen al área laboral pública $(51,4 \%)$ y tienen más de 10 años de experiencia laboral $(41,4 \%)$.

Percepción del estrés por el trabajo a distancia (EST) (Tabla 2): En el ambiente actual de aislamiento por el COVID-19, se puede observar que los promedios apuntan a que los trabajadores se perciben tensos o preocupados y ansiosos al realizar sus actividades de trabajo desde sus hogares.

Tabla 1. Características sociodemográficas y laborales de trabajadores en teletrabajo, septiembre-octubre 2020 $(\mathrm{n}=181)$.

\begin{tabular}{llrc}
\hline Variables sociodemográficas & & $\mathbf{f r}$ & $\mathbf{\%}$ \\
\hline Género & Masculino & 43 & $23,8 \%$ \\
& Femenino & 138 & $76,2 \%$ \\
\hline Estado Civil & Soltero & 101 & $55,8 \%$ \\
& Casado & 65 & $35,9 \%$ \\
& Prefiero no decirlo & 15 & $8,3 \%$ \\
\hline Año de Nacimiento & Antes de 1963 & 26 & $14,4 \%$ \\
& De 1963 a 1980 & 54 & $29,8 \%$ \\
& De 1981 a 1997 & 96 & $53,0 \%$ \\
& Después de 1997 & 5 & $2,8 \%$ \\
\hline Número de hijos & No tiene hijos & 81 & $44,8 \%$ \\
& Un hijo & 28 & $15,5 \%$ \\
& Dos hijos & 44 & $24,3 \%$ \\
& Tres hijos & 26 & $14,3 \%$ \\
& Más de tres & 2 & $1,1 \%$ \\
\hline Región en la que vive & Región IV & 9 & $5,0 \%$ \\
& Región VIII & 15 & $8,3 \%$ \\
& Región XIII & 35 & $19,3 \%$ \\
& Región XVI & 109 & $60,2 \%$ \\
& Otras regiones & 13 & $7,2 \%$ \\
\hline
\end{tabular}




\section{Continuación Tabla 1}

\begin{tabular}{llcc}
\hline Variables sociodemográficas & & fr & \% \\
\hline Rubro empresa & Educación & 67 & $37,0 \%$ \\
& Salud & 28 & $15,5 \%$ \\
& Servicios & 27 & $14,9 \%$ \\
& Comercial & 19 & $10,5 \%$ \\
& Sector público/ Gubernamental & 26 & $14,4 \%$ \\
& Industrial/ Manufactura & 9 & $5,0 \%$ \\
& Otro & 5 & $2,8 \%$ \\
\hline Tipo de institución en la que trabaja a a blica & 93 & $51,4 \%$ \\
& Privada & 88 & $48,6 \%$ \\
\hline Años de experiencia laboral & Menos de 1 año & 23 & $12,7 \%$ \\
& Entre 1 y 5 años & 53 & $29,3 \%$ \\
& Entre 6 y 10 años & 30 & $16,6 \%$ \\
& Más de 10 años & 75 & $41,4 \%$ \\
\hline
\end{tabular}

Tabla 2. Percepción del estrés por el trabajo a distancia en el entorno de COVID-19, septiembre-octubre 2020 $(\mathrm{n}=181)$.

\begin{tabular}{|c|c|c|c|c|c|c|c|c|}
\hline \multirow{2}{*}{$\begin{array}{l}\text { Percepción del estrés por el trabajo a distancia en } \\
\text { el entorno de COVID-19 }\end{array}$} & \multicolumn{2}{|c|}{$\begin{array}{c}\text { Desacuerdo } \\
1-2\end{array}$} & \multicolumn{2}{|c|}{$\begin{array}{l}\text { Indiferente } \\
\quad 3\end{array}$} & \multicolumn{2}{|c|}{$\begin{array}{l}\text { Acuerdo } \\
4-5\end{array}$} & \multirow[t]{2}{*}{ MD } & \multirow[t]{2}{*}{ DS } \\
\hline & Fr & $\%$ & Fr & $\%$ & Fr & $\%$ & & \\
\hline $\begin{array}{l}\text { a. Estoy tenso o preocupado por realizar mis } \\
\text { actividades de trabajo desde la casa (EST1) }\end{array}$ & 49 & 27,1 & 43 & 23,8 & 89 & 49,1 & 3,29 & 1,33 \\
\hline $\begin{array}{l}\text { b. Siento ansiedad (nerviosismo) por hacer mis } \\
\text { actividades de trabajo desde la casa (EST2) }\end{array}$ & 58 & 32,0 & 43 & 23,8 & 80 & 44,2 & 3,13 & 1,37 \\
\hline $\begin{array}{l}\text { c. Siento enojo por estar realizando mis actividades } \\
\text { de trabajo desde la casa (EST3) }\end{array}$ & 111 & 61,3 & 36 & 19,9 & 34 & 18,8 & 2,23 & 1,27 \\
\hline $\begin{array}{l}\text { d. Estoy tenso o preocupado por los gastos que se } \\
\text { generan al trabajar desde casa (EST4) }\end{array}$ & 112 & 61,9 & 32 & 17,7 & 37 & 20,4 & 2,25 & 1,27 \\
\hline
\end{tabular}

MD: Promedio; DS: Desviación Estándar.

Actitudes ante el cambio por realizar teletrabajo por el COVID-19 (Tabla 3): Se observó que las principales actitudes de los trabajadores ante el cambio de realizar teletrabajo debido a la pandemia corresponden a tratar de vivir el presente, seguido por tratar de buscar la manera de ayudar a los demás.

Correlación entre las variables de la investigación (Tabla 4): Se pudo determinar que el Estrés por el trabajo a distancia y el Balance Vida-Trabajo presentan una correlación inversa de $-0,408(\mathrm{p}<0,01)$, por lo tanto, a menor estrés por el trabajo a distancia, sería mayor el Balance Vida-Trabajo. Referente a los ítems de Teletrabajo (que considera aspectos de condiciones generales, procesos, infraestructura) y Balance Vida-Trabajo, también tienen una correlación positiva de 0,276 $(\mathrm{p}<0,01)$, en tanto a condiciones favorables para la realización de Teletrabajo, mayor es la percepción de Balance Vida-Trabajo. En cambio, la correlación entre Teletrabajo y Estrés por el trabajo a distancia, es inversa, $-0,373(\mathrm{p}<0,01)$, luego a condiciones favorables para la realización de Teletrabajo, menor es la percepción de Estrés por el trabajo a distancia. 
Tabla 3. Actitudes ante el cambio por realizar teletrabajo en el entorno de COVID-19, septiembre-octubre 2020 $(\mathrm{n}=181)$.

\begin{tabular}{|c|c|c|c|c|c|c|c|c|}
\hline \multirow{2}{*}{$\begin{array}{l}\text { Actitudes ante el cambio por realizar teletrabajo } \\
\text { en el entorno de COVID-19 }\end{array}$} & \multicolumn{2}{|c|}{$\begin{array}{c}\text { Desacuerdo } \\
1-2\end{array}$} & \multicolumn{2}{|c|}{$\begin{array}{l}\text { Indiferente } \\
\quad 3\end{array}$} & \multicolumn{2}{|c|}{$\begin{array}{c}\text { Acuerdo } \\
4-5\end{array}$} & \multirow[t]{2}{*}{ MD } & \multirow[t]{2}{*}{ DS } \\
\hline & Fr & $\%$ & $\mathrm{Fr}$ & $\%$ & Fr & $\%$ & & \\
\hline $\begin{array}{l}\text { a. Me molesto fácilmente, soy poco tolerante ante } \\
\text { la situación }\end{array}$ & 82 & 45,3 & 53 & 29,3 & 46 & 25,4 & 2,71 & 1,14 \\
\hline $\begin{array}{l}\text { b. Estoy desesperado por adquirir mis bienes de } \\
\text { consumos (realizar compras de pánico) }\end{array}$ & 139 & 76,8 & 23 & 12,7 & 19 & 10,5 & 1,88 & 1,09 \\
\hline $\begin{array}{l}\text { c. Dejo de consumir lo que me hace dańo (desde } \\
\text { alimentos hasta noticias) }\end{array}$ & 37 & 20,4 & 50 & 27,7 & 94 & 51,9 & 3,49 & 1,28 \\
\hline $\begin{array}{l}\text { d. Considero que se está tratando de hacer lo mejor } \\
\text { ante la situación complicada }\end{array}$ & 34 & 18,7 & 41 & 22,7 & 106 & 58,6 & 3,65 & 1,28 \\
\hline $\begin{array}{l}\text { e. Pienso en los demás y busco la manera de } \\
\text { ayudarlos }\end{array}$ & 6 & 3,3 & 37 & 20,4 & 138 & 76,3 & 4,08 & 0,83 \\
\hline $\begin{array}{l}\text { f. Trato de vivir el presente, día a día de acuerdo a } \\
\text { las circunstancias }\end{array}$ & 9 & 5 & 34 & 18,7 & 138 & 76,3 & 4,16 & 0,94 \\
\hline
\end{tabular}

MD: Promedio; DS: Desviación Estándar.

Tabla 4. Correlación entre Estrés por el trabajo a distancia, Balance vida-trabajo, Teletrabajo y Satisfacción con el teletrabajo.

\begin{tabular}{l|l|l|l|l|l}
\hline \multicolumn{2}{l|}{} & BVT & EST & TET & SAT \\
\hline BVT & Coef. Pearson & 1 & & & \\
& Sig. & & & & \\
& $\mathrm{N}$ & 181 & & & \\
\hline \multirow{2}{*}{ EST } & Coef. Pearson & $-0,408^{* *}$ & 1 & & \\
& Sig. & 0 & & & \\
& $\mathrm{~N}$ & 181 & 181 & & \\
\hline TET & Coef. Pearson & $0,276^{* *}$ & $-0,373^{* *}$ & 1 & \\
& Sig. & 0 & 0 & & \\
& $\mathrm{~N}$ & 181 & 181 & 181 & \\
\hline SAT & Coef. Pearson & $0,205^{* *}$ & $-0,287^{* *}$ & $0,381^{* *}$ & 1 \\
& Sig. & 0,006 & 0 & 0 & \\
& $\mathrm{~N}$ & 181 & 181 & 181 & 181 \\
\hline
\end{tabular}

${ }^{*}$ Nivel de significancia menor de 0.05

${ }^{* *}$ Nivel de significancia menor de 0.01

Finalmente, la correlación entre la Satisfacción con el teletrabajo y el Balance Vida-Trabajo es directa, de modo que, ante mayor Satisfacción con el teletrabajo, mayor es el Balance Vida-Trabajo $(\mathrm{p}<0,01)$ y se aprecia una correlación inversa de $-0,287(\mathrm{p}<0,01)$ entre Satisfacción con el teletrabajo y Estrés por el trabajo a distancia, en tanto, a mayor satisfacción con el teletrabajo, menor es la percepción de estrés.

\section{DISCUSIÓN}

Por la presencia del COVID-19, el teletrabajo desde casa ha implicado una diversidad de cambios en las formas de realizar el trabajo y ha estado generando una serie de reflexiones y aprendizajes en el corto y largo plazo. Al parecer ha llegado para quedarse y muchas organizaciones están reaccionando con esta modalidad para seguir adelante en el mercado. 
Este estudio determinó que los trabajadores se reportan tensos, preocupados o ansiosos en la realización de las actividades telemáticas. Presentan sentimientos y emociones con promedios que permiten observar factores generadores de estrés tal como lo planteado por Madero y Flores ${ }^{(20)}$. Sumado a esto, Madero ${ }^{(19)}$ señala que realizar el trabajo a distancia es causa de "preocupación, ansiedad y molestia", que pueden transformarse en fuentes generadoras de estrés, impactando en la salud de los trabajadores, y consecuentemente en sus familiares o personas con las que convive. En este mismo contexto, Tavares ${ }^{(8)}$ identificó diferentes problemas relacionados con el teletrabajo, entre ellos el estrés relacionado con: el exceso de trabajo, plazos ajustados, horas de trabajo muy largas, incapacidad de desconexión y menos tiempo para descansar. En la misma línea, un estudio chileno determinó que el aislamiento debido al TET puede tener un potencial efecto negativo sobre la salud ocupacional y el bienestar, y que los altos niveles de intensidad laboral y los conflictos que se pudieran generar entre el tiempo dedicado al trabajo y a la vida familiar están asociados con estrés en el trabajo y con efectos negativos en la salud y el bienestar de los trabajadores ${ }^{(7)}$. Asimismo, Martin $^{(27)}$ postula que la continua disponibilidad del trabajador para solventar cuestiones laborales supone una fuente generadora de estrés, causado, muchas veces, por la falta de habilidad para tratar las tecnologías del ordenador de manera saludable, lo que conlleva al uso excesivo y la falta de desconexión digital. En este sentido, un estudio asiático acuñó el termino "techno estrés", para referenciar al estrés ocasionado por esta modalidad laboral y sus resultados mostraron que la tecnología y las características de trabajo inducen al techno estrés en los teletrabajadores ${ }^{(28)}$.

Por otra parte, frente a las actitudes ante el cambio por realizar teletrabajo por el COVID-19, se observaron resultados interesantes, relevando actitudes positivas, donde los trabajadores optan por vivir el presente diariamente, de acuerdo a las circunstancias e incluso buscar modos de ayuda a los demás. Asimismo, consideran que se está tratando de hacer lo mejor ante la situación complicada que se está viviendo. En este contexto, Jiménez ${ }^{(29)}$ señala que la actitud positiva frente a los problemas ayuda a generar motivación y estimula la resistencia a la adversidad. Para mantener esta actitud y pensamientos positivos, se debe disfrutar de momentos de bienestar ${ }^{(29)}$, incorporar en la rutina diaria tiempos para realizar actividades relajantes y placenteras, asimismo, conservar una actitud positiva frente a lo que está ocurriendo y mantenerse mentalmente activo puede ayudar a sobrellevar este desafío con mayor bienestar emocional $^{(30)}$.

Utilizando como base la propuesta que hace $\mathrm{Hemmi}^{(31)}$ sobre la actitud de las personas mostrada ante los cambios en el entorno, se podrían mencionar algunas categorías que, de alguna forma, pudieran reflejar las actitudes emocionales que manifiestan las personas ante esta situación del COVID-19. Se observa una percepción de actitudes de superación, donde el trabajador toma control emocional de sí mismo y puede distinguir con claridad su propia interacción con otros sujetos y medios de comunicación. De igual modo, al indagar qué es lo mejor que está tratando de hacer ante lo complicado que es esta situación, se aprecia en los trabajadores la percepción de actitudes emprendedoras (zona de aprendizaje) porque aprenden a distinguir la información que produce un estado optimista de aquella que altera el estado de ánimo. Vinculado a esto, las actitudes ante el cambio con menor promedio estarían relacionadas con la situación emocional, que Hemmi la identifica como una zona de pánico, en la que se focalizan irritabilidad, menor confianza o compras de pánico, por ejemplo, como las realizadas para prevenir los efectos de la pandemia ${ }^{(31)}$.

Considerando los resultados de la Tabla 4, destaca que el BVT mejora cuando el trabajador percibe menos estrés, observa condiciones favorables para la realización del teletrabajo y tiene mayor satisfacción con su actividad. En este sentido, diversos autores se han referido al tema del BVT durante el teletrabajo: Briones y Alverca ${ }^{(32)}$, en Guayaquil, indican que debido a la extensión de los horarios, la modalidad telemática no permite a los trabajadores compartir en ciertas cotidianidades con sus familias, por las múltiples responsabilidades laborales que deben cumplir; Martín ${ }^{(27)}$ concluye que puede existir una interferencia entre la vida personal y laboral porque desaparecen los límites entre el tiempo de trabajo y el tiempo de descanso o libre. Aun así, se plantea que tiene efectos positivos en la dinámica familiar-laboral ${ }^{(8)}$, principalmente por la flexibilidad y autonomía de esta modalidad ${ }^{(5)}$, 
que permiten organizar las actividades profesionales y domésticas y con ello conciliar la vida laboral y familiar ${ }^{(9)}$.

Ahora bien, como se apreció en los resultados, las condiciones en que se realiza el Teletrabajo juegan un rol importante, porque si son favorables cobran relevancia a la hora de mantener este Bienestar vida-trabajo y disminuir la percepción de estrés. $\mathrm{Si}$ en época de contingencia el trabajador tiene condiciones adecuadas para realizar el trabajo a distancia, se le facilitan los procesos para enfrentar la actividad telemática y cuenta con la infraestructura adecuada, sin lugar a dudas podrá realizar su actividad laboral con menores inconvenientes, percibirá menor estrés por el trabajo a distancia y mayor satisfacción.

Respecto de la satisfacción con el teletrabajo, el Balance Vida-Trabajo, y la percepción de estrés por el trabajo a distancia, se observó que ante una mayor satisfacción con el teletrabajo, mayor es el Balance Vida-Trabajo y menor es la percepción de estrés. Al respecto, Jiménez y Moyano ${ }^{(33)}$ señalan que aplicar medidas de "política organizacional" como "flexibilidad horaria, permisos, asistencia en labores domésticas", entre otros, generan un aumento de la satisfacción laboral y disminuyen los niveles de estrés, además permite un mayor "control personal del trabajo y del tiempo familiar". Por su parte, Chiang et al. ${ }^{(34)}$ determinaron en sus estudios que, "a mayor percepción de las variables de Satisfacción laboral y mayor Control sobre el trabajo, se logra reducir el grado de estrés". En este mismo contexto, en un estudio realizado en Hong Kong, que analizó la satisfacción laboral en Teletrabajadores, se concluyó que "la tecnología y las características de trabajo inducen al techno estrés en los teletrabajadores", lo que podría reducir la satisfacción laboral dependiendo de la intensidad del teletrabajo ${ }^{(28)}$.

Todo lo descrito invita a reflexionar respecto de la relevancia que tienen algunas prácticas en materia de recursos humanos y sus implicaciones en la salud física y emocional de las personas, en especial en el contexto del teletrabajo. Antes de la pandemia, esta modalidad era vista como un incentivo para las personas o como un mecanismo de distinción ${ }^{(35)}$, sin embargo, ahora es una alternativa laboral para todos y un factor diferenciador en la búsqueda de algún empleo. Por el contexto en el que se está realizando, es probable que se siga manteniendo por más tiempo mientras no se alcancen las cifras de inmunización necesarias. Por ello es necesario seguir midiendo los efectos del teletrabajo, no solo en el área de la salud mental, también en la salud física.

Para una adecuada aplicabilidad de esta modalidad, es prioritario el apoyo de las jefaturas en beneficio de facilitar aquellos procesos organizacionales que otorguen las condiciones apropiadas para que el trabajador cuente con la infraestructura y la tecnología requerida.

Respecto de las limitaciones de este estudio, se puede señalar que, por el tipo de muestreo, aun cuando se alcanzó un adecuado tamaño de muestra, los resultados no pueden ser generalizados a toda la población de trabajadores que realizan teletrabajo. No obstante, los resultados contribuyen a abrir líneas investigativas en el tema del teletrabajo para ir evidenciando sus efectos en la salud de los trabajadores, más aún cuando esta modalidad se ha prolongado más de lo que se pensó en sus inicios y en algunas empresas se comenzará a adoptar permanentemente, ya sea como modalidad mixta o exclusiva en algunos puestos de trabajo.

\section{CONCLUSIONES}

Los resultados de este estudio permiten concluir que los trabajadores que se encuentran en modalidad telemática, corresponden mayormente a mujeres, solteras, entre 24 a 40 años, que se desempeñan en el ámbito educativo, del sector público y tienen experiencia laboral mayor a diez años.

Asimismo, las actividades telemáticas producen en los trabajadores sintomatología derivada del estrés, como tensión y/o ansiedad, pero a pesar de esto, tienen una actitud positiva ante este cambio en la modalidad laboral, tratando de vivir el día a día y buscando cómo apoyar a otros.

Los trabajadores que perciben mayor estrés por el trabajo a distancia presentan un menor balance entre sus actividades personales y laborales y una menor satisfacción por las actividades que realiza.

El presentar condiciones favorables para la realización del teletrabajo disminuye la percepción de estrés y contribuye a la conciliación entre la vida familiar y laboral, además de incrementar la satisfacción con las actividades telemáticas.

Con estos resultados se espera que los res- 
ponsables de las áreas de recursos humanos de las empresas puedan diseñar y desarrollar estrategias relacionadas con el bienestar integral de las personas y fomentar acciones que ayuden a mejorar la salud emocional de sus colaboradores. Es necesario incorporar prácticas de gestión que favorezcan ese balance entre las actividades laborales y familiares. Así también, a los trabajadores que realizan actividades telemáticas se les debe motivar a realizar ejercicio físico o actividades de distracción para ayudar a su desarrollo personal.

\section{REFERENCIAS}

1. Alzúa M.L, Gosis P. Impacto Social y Económico de la COVID-19 y Opciones de Políticas en Argentina [Internet]. Nueva York: PNUD; 2020 [citado 31 jul 2020]. Disponible en: https://www. latinamerica.undp.org/content/rblac/es/home/ library/covid-19--policy-papers.html

2. Ozamiz N, Dosil M, Picaza M, Idoiaga N. Niveles de estrés, ansiedad y depresión en la primera fase del brote del COVID-19 en una muestra recogida en el norte de España. Cad Saúde Pública [Internet]. 2020 [citado 31 jul 2020]; 36(4): e00054020. Disponible en: https://doi.org/10.1590/0102-311 X00054020

3. Santillán W. El teletrabajo en el COVID-19. CienciAmérica [Internet]. 2020 [citado 31 jul 2020]; 9(2): 65-76. Disponible en: http://dx.doi. org/10.33210/ca.v9i2.289

4. Perticará $M$, Tejada M. Sobre vulnerabilidad y teletrabajo durante la pandemia. Observatorio Económico [Internet]. 2020 [citado 31 jul 2020]; 144: 4-5. Disponible en: https://doi.org/10.11565/ oe.vi144.365

5. Vicente $\mathrm{M}$, Torres I, Torres A, Ramírez M, Capdevila L. El teletrabajo en salud laboral: Aspectos médico-legales y laborales. CES Derecho [Internet]. 2018 [citado 31 jul 2020]; 9 (2): 287297. Disponible en: https://www.researchgate.net/ publication/330555210

6. Asociación Chilena de Seguridad. Aspectos legales en trabajo a distancia y teletrabajo. ACHS Ficha Técnica [Internet]. 2020 [citado 25 abr 2020]. 2 p. Disponible en: https://www.achs.cl/portal/centrode-noticias/Documents/Aspectos-legales-trabajo-adistancia.pdf

7. Lampert MP, Poblete M. Efectos positivos y negativos del teletrabajo sobre la salud. Biblioteca del Congreso Nacional de Chile [Internet]. 2018 [citado 31 jul 2020]. Sup 117515.9 p. Disponible en: https://obtienearchivo.bcn.cl/obtienearchivo? $\mathrm{id}=$ repositorio/10221/26041/1/BCN_Efectos_ del_teletrabajo_sobre_la_salud_FINAL.pdf

8. Tavares A. Telework and health effects review. International Journal of Healthcare [Internet]. 2017 [citado 21 jul 2020]; 3(2): 30-36. Disponible en: $10.5430 /$ ijh.v3n2p30

9. Martín P. Teletrabajo y comercio electrónico [Internet]. España: Ministerio de Educación, Cultura y Deporte; 2018 [citado 21 jul 2020]; 3(2). Disponible en: https://sede.educacion.gob.es/ publiventa/PdfServlet?pdf=VP18905.pdf\&area=E

10. Greenhaus JH, Collins KM, Shaw JD. The relation between work-family balance and quality of life. J Vocat Behav [Internet]. 2003 [citado 2 mar 2021]; 63 (3): 510-31. Disponible en: https://doi. org/10.1016/S0001-8791(02)00042-8

11. Muñoz-Araya V, Mayta-Tristán P. Balance trabajo-vida: un nuevo indicador del bienestar de profesionales de la salud. Rev méd Chile [Internet]. 2017 Oct [citado 2 mar 2021]; 145(10): 13611362. Disponible en: http://dx.doi.org/10.4067/ S0034-98872017001001361

12. Greenhaus JH, Beutell NJ. Sources of conflict between work and family roles. Acad Manage Rev [Internet]. 1985 [citado 5 may 2021]; 10(1): 76-88. Disponible en: https://doi.org/10.5465/ amr.1985.4277352

13. Luengo C, Martínez A, Muñoz N, Pino A, Palma M. Conflicto Trabajo-Familia en el Equipo de Enfermería de un Hospital Clínico de Alta Complejidad en Chile, 2018. GJMR-K [Internet]. 2020 [citado 5 mar 2021]; 20(2). Disponible en: https://medicalresearchjournal.org/index.php/ GJMR/article/view/2004

14. Bae K, Yang G. The Effects of Family-Friendly Policies on Job Satisfaction and Organizational Commitment. PPM [Internet]. 2017 [citado 31 jul 2020]; 46 (1). Disponible en: https://doi. org/10.1177/0091026016689669

15. Acuña M, Lema MJ, Luengo C. Asociación entre factores sociodemográficos, laborales y de salud con la percepción de estrés laboral en profesionales de la salud. rev cuba salud trabajo [Internet]. 2021 [citado 6 sep 2021]; 22(1): [aprox. 7 p.]. Disponible en: http://revsaludtrabajo.sld.cu/index.php/revsyt/ article/view/186

16. Breso E, Caballero C, González O. Burnout en estudiantes universitarios. Psicol desde el Caribe [Internet]. 2015 [citado 6 sep 2021]; 32(3): 424441. Disponible en: http://dx.doi.org/10.14482/ psdc.32.3.6217

17. Soto T, Vera C, Fuenzalida J, Díaz R, Darville P. Teletrabajo en el Estado de Chile: Efectos y desafíos para su diseño e implementación [Internet]. 
Universidad de Chile. Chile: Facultad de Ciencias Físicas y Matemáticas; 2018 [citado 6 mar 2021]. Disponible en: http://www.trendtic.cl/wp-content/ uploads/2018/10/CSP-SSP16-Teletrabajo-en-elEstado-de-Chile-1.pdf

18. Centro de Estudio del Trabajo y Factores Humanos. Guía para el teletrabajo en condiciones de pandemia COVID-19 [Internet]. Universidad de Valparaíso. 2020 [citado 10 jun 2020]. 8 p. Disponible en: https://bibliotecas.uv.cl/images/Dibra/ Coronavirus/guia_teletrabajo_en_pandemia_ covid19.pdf

19. Madero S, Ortiz O, Ramírez J, Olivas M. Stress and myths related to the COVID-19 pandemic's effects on remote work. Management Research [Internet]. 2020 [citado 6 sep 2021]; 18(4): 401420. Disponible en: https://doi.org/10.1108/ MRJIAM-06-2020-1065

20. Madero S, Flores R. Predictores de la disposición de trabajadores mexicanos a aceptar el teletrabajo. Investigación y Ciencia [Internet]. 2009 [citado 6 sep 2021]; 43(17): 46-52. Disponible en: https:// www.redalyc.org/articulo.oa?id=67411387009

21. Madero S, Flores R. Forzados a trabajar a distancia por el virus A H1N1: Exploración del estrés por el trabajo a distancia y uso de tecnología, con la satisfacción con el trabajo y la disposición a realizar actividades a distancia. XIV Congreso Internacional de Investigación en Ciencias Administrativas ACACIA. Realizado en EGADE Business School. 2010. Monterrey NL: Instituto Tecnológico y de Estudios Superiores de Monterrey.

22. Ministerio de Salud. Sobre la investigación Científica en el ser humano, su genoma, y prohíbe la Clonación humana. Ley 20.120 [Internet]. Subsecretaría de Salud Pública. [citado 1 ago 2020]. 4 p. Disponible en: http://bcn.cl/2fe0y

23. Ministerio de Salud. Sobre la protección de la vida privada. Ley 19.628 [Internet]. Subsecretaría de Salud Pública. [citado 1 ago 2020]. 13 p. Disponible en: http://bcn.cl/2f7cg

24. Arroyo F. El código de Nuremberg: Un hito en la ética de la investigación médica. Rev Fac Cien Med [Internet]. 1999 [citado 5 ago 2020]; 24(1): 31-5. Disponible en: https://revistadigital.uce.edu. ec/index.php/CIENCIAS_MEDICAS/article/ view/873

25. Rodríguez E. Comités de evaluación ética y científica para la investigación en seres humanos y las pautas CIOMS 2002. Acta bioeth [Internet]. 2004 [citado 5 ago 2020]; 10(1): 37-48. Disponible en: http:// dx.doi.org/10.4067/S1726-569X2004000100005

26. Celina H, Campo A. Aproximación al uso del coeficiente alfa de Cronbach. Rev colomb psiquiatr [Internet]. 2005 [citado 5 mar 2021]; XXXIV(4):
572-580. Disponible en: https://www.redalyc.org/ articulo.oa?id $=80634409$

27. Martin O. El tecnoestrés como factor de riesgo para la seguridad y salud del trabajador. LAN Revista de relaciones laborales [Internet]. 2021 [citado 6 sep 2021]; 44. Disponible en: https://doi.org/10.1387/ lan-harremanak.22239

28. Suh A, Lee J. Understanding teleworkers' technostress and its influence on job satisfaction. Internet Research [Internet]. 2017 [citado $20 \mathrm{dic}$ 2020]; 27(1): 140-159. Disponible en: https://doi. org/10.1108/IntR-06-2015-0181

29. Jiménez L. Afrontamiento emocional para los trabajadores de la salud ante pandemia del COVID-19. Rev med Costa Rica [Internet]. 2020 [citado 6 mar 2021]; 85(629). Disponible en: http://revistamedicacr.com/index.php/rmcr/ article/view/285

30. Ministerio de Salud. Guía Práctica de Bienestar Emocional Cuarentena en tiempo de COVID-19 [Internet]. 2021 [citado 6 sept 2020]. 36 p. Disponible en: https://www.minsal.cl/wp-content/up loads/2020/04/2020.04.12_GUIA-PRACTI CA-CUARENTENA-EN-TIEMPOS-DECOVID19_final.pdf

31. Hemmi M. ¿Te atreves a Soñar? $1^{a}$ edición. Argentina: Grijalbo; 2016.

32. Briones R, Alverca R. Las percepciones de los trabajadores frente al teletrabajo durante la pandemia Covid-19, caso de estudio realizado a los residentes de una urbanización del Cantón Daule [Tesis doctoral]. Guayaquil, Ecuador: Universidad Católica de Santiago de Guayaquil; 2020. Disponible en: http://repositorio.ucsg.edu. ec/handle/3317/15593

33. Jiménez A, Moyano E. Factores laborales de equilibrio entre trabajo y familia: medios para mejorar la Calidad de Vida. Universum [Internet]. 2008 [citado 6 sept 2021]; 23(1): 116-133. Disponible en: https://dx.doi.org/10.4067/S071823762008000100007

34. Chiang M, Riquelme R, Rivas A. Relación entre Satisfacción Laboral, Estrés Laboral y sus Resultados en Trabajadores de una Institución de Beneficencia de la Provincia de Concepción. Cienc Trab [Internet]. 2018 [citado 06 sep 2021]; 20(63): 178-186. Disponible en: http://dx.doi. org/10.4067/S0718-24492018000300178

35. Madero S. Modelo de Retención Laboral de Millennials, desde la Perspectiva Mexicana. Investig adm [Internet]. 2019 [citado 6 sep 2021]; 48(124). Disponible en: http://www.scielo.org. $\mathrm{mx} /$ scielo.php?script $=$ sci_arttext $\&$ pid $=\mathrm{S} 2448$ $76782019000200001 \& \operatorname{lng}=$ es\&nrm=iso 